\title{
MEDICAL IMAGE FUSION OF MULTI MODAL IMAGES USING RANDOM BLOCK SELECTION METHOD
}

\author{
D. Sheefa Ruby Grace ${ }^{\# 1}$, Dr. Mary Immaculate Sheela ${ }^{* 2}$ \\ \# Research Scholar, Bharathiar University, Coimbatore, India \\ ${ }^{1}$ samsheef@gmail.com \\ * Research Supervisor, Bharathiar University, Coimbatore, India \\ 2drsheela09@gmail.com
}

\begin{abstract}
Image fusion is a process of obtaining a single image by combining multiple images. Since the source images are obtained from various detectors at dissimilar times, some information will be missing in one source image and that missing information may present in another source image. Hence the physicians are in the need of combining the fine information from both source images into a single one.

Objective: The objective of image fusion is providing utmost information that is missing in the source images.

Method: In this paper CT (Computed Tomography) and PET (Positron Emission Tomography) images are get fused. The CT imaging provides anatomic information whereas the PET image provides functional information of the body. The proposed method fused the images using contourlet based random block selection method with MAX fusion rule.
\end{abstract}

Result: It is proved that the proposed method provides better result with less number of computations. Experimental results are taken by writing MATLAB code. To prove the quality of fused image, performance measures such as Peak Signal to Noise Ratio (PSNR), Structural Similarity Index Measure (SSIM) and Mutual Information (MI) are used.

Keywords: CT, PET, image fusion, contourlet

\section{INTRODUCTION}

Image fusion combines registered images into a single composite image so as to maximize relevant information and quality. The fused image will improve the performance of image analysis algorithms used in different applications. Different medical imaging modalities such as Computed Tomography (CT), Magnetic Resonance Imaging (MRI),Positron Emission Tomography (PET), Single Photon Emission Tomography (SPECT) delivers different useful information about the human body which helps doctors to diagnose diseases. However, these modalities are not capable of providing complete information by observational limitations [1]. CT provides information regarding dense structures like bones while information regarding soft tissues can be obtained by using MRI and PET reveals the anatomic structure of organ or a tissue. Hence a good fusion algorithm is needed to produce a single image to provide more accurate diagnoses.

Image fusion techniques are classified into pixel and feature points. Pixel fusion works directly on the pixels of source images while feature fusion techniques function on features taken out from the source images. Pixel level fusion leads to unwanted side effects such as reduced contrast [2]. In Recent years, various methods have been developed to perform image fusion. Some well-known image fusion methods are, Intensity-huesaturation (IHS) transform based fusion, Principal component analysis (PCA) based fusion, Pyramid method, Wavelet transform, Multi-wavelet transform, curvelet transform and contourlet transform [3].

Before implementing the proposed method, some of the existing research works have been analysed. A novel Non Sub sampled Contourlet Transform (NSCT) based method for multimodal medical image fusion algorithm was proposed by Yang et al., in which the phase congruency and Log-Gabor energy are integratedly used as the fusion rules for low and high-frequency coefficients, respectively [4].A new algorithm was developed by Venkataraman et al. which employ PCA and Sparse representation for common and innovative sparse components of the source images [5]. Sudeb and Malay implemented a novel approach to the multimodal medical image fusion (MIF) problem, employing multiscale geometric analysis of non-subsampled contourlet transform and fuzzy-adaptive reduced pulse-coupled neural network (RPCNN) [6].A pixel level based hybrid concept has proposed to fuse multimodal CT and PET images by GuruPrasad et al. It integrates the conventional and advance fusion methods to overcome their demerits and enhance the image processing qualities like Principal Component Analysis (PCA), Discrete Wavelet Transform (DWT) and Discrete Curvelet Transform (DCT) using one DWT-DCT-PCA algorithm [7]. Even though many methods are available for image fusion, 
each method is having some drawbacks. The proposed method provided better results comparing to the existing fusion methods.

\section{MATERIALS AND METHODS}

In the proposed method, two different multi modal medical images CT and PET are used as the source images. Initially, the two source images are divided into smaller blocks of size mxn. Then, each block from both input images are compared for similarity using the Structure Similarity Measure (SSIM). If they are likewise, then there is no need of fusion process. Select any one of the block using random block selection method (RBS) and copy the selected block into resultant fused block. Otherwise, the blocks are decomposed into coefficients using contourlet transform. Contourlet transform is more accurate to deal with the curve than wavelet transform [8].MAX fusion rule is applied to select the best coefficient. Finally, inverse contourlet transform is applied to get the fused image. An outline of the proposed method is given in fig.1

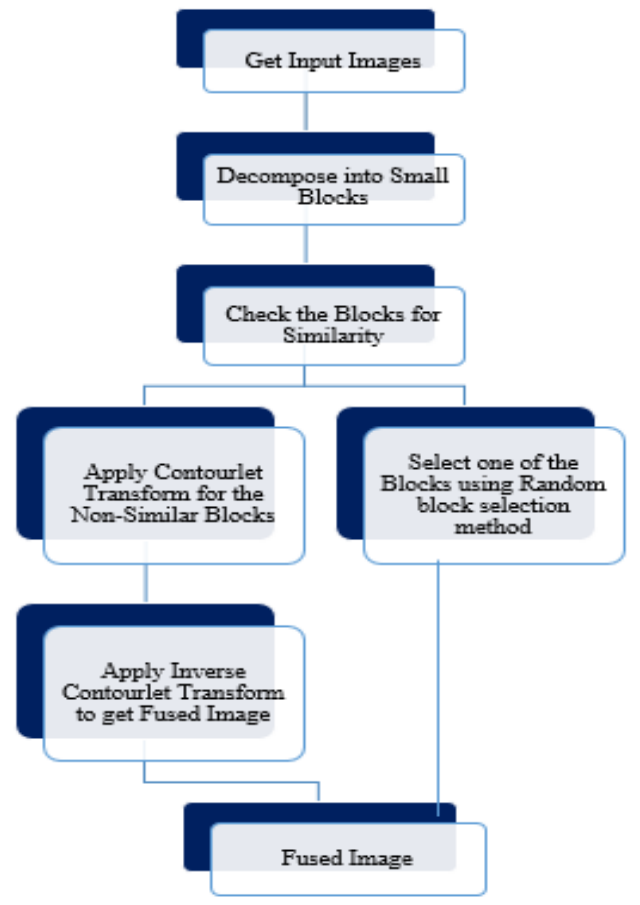

Fig.1 Outline of Proposed Method

The stepwise detailed algorithm of the proposed method is explained as follows

Input :Two input images CT and PET of same size

Output: A single fused image, FI

Step 1. Read the two input images, Y1 and Y2 to be fused.

Step 2. Divide both input images into smaller blocks of size mxn

Step 3. Compare the $i^{\text {th }}$ block of both input images by calculating SSIM

Step 4. If the value of SSIM $>0.9$ then

i) Both blocks are the same

ii) No need of applying contourlet transform

iii) Select any block randomly (RBS) and copy to the fused block

Step 5. Else apply the contourlet transform to the blocks

i) Decompose them into coefficients

ii) Apply MAX fusion rule to choose the best coefficient

iii) Apply Inverse Transform to the coefficients to get the fused block

Step 6: Repeat the process from Step 3 for all the blocks of the input images

Since the selective blocks only are processed by contourlet transform in the proposed method, the number of computations is minimized. It is proved that the proposed method obtained the good quality of fused image with minimum number of computations comparing to the existing methods. 


\section{RESULT AND DISCUSSION}

Image fusion is performed on the two different multi modal images, CT and PET. The proposed method is implemented using MatLab. When the reference image is not available, the performance of the resultant fused image can be evaluated by the non-reference quality metrics such as Feature Mutual Information (FMI) and Entropy whereas Peak Signal to Noise Ratio (PSNR), Structural Similarity Index Measure (SSIM) can be used when reference image is existing[9]. The source images are taken from Osirix database which contains Dicom images with .dcm extension acquired by the equipment CT and PET [10].

\section{A. Feature Mutual Information (FMI)}

The amount of feature information, which the fused image $\mathrm{F}$ contains about the input images A and $\mathrm{B}$, is exclusively measured by means of MI using the following equation 1 ,

$$
\begin{aligned}
& I_{F A}=\sum_{f, a} P_{F A}(x, y, z, w) \log _{2} \frac{P_{F A}(x, y, z, w)}{P_{F}(x, y) \cdot P_{A}(z, w)} \\
& I_{F B}=\sum_{f, b} P_{F B}(x, y, z, w) \log _{2} \frac{P_{F B}(x, y, z, w)}{P_{F}(x, y) \cdot P_{B}(z, w)}
\end{aligned}
$$

And the FMI metric is

$$
F M I=I_{F A}+I_{F B}
$$

B. Entropy

The amount of information present in an image is measured by entropy. The fused image which contains the maximum of Entropy will give the best fusion technique. Entropy can be calculated using the equation 2 .

$$
H=-\sum_{i=0}^{L-1} h_{F}(i) \log _{2} h_{F}(i)
$$

The values of non-reference quality measures FMI and Entropy are summarized in Table I. The proposed RBS method produced higher values than the existing method. Not all the blocks of input images are get processed for fusion in the proposed method, but the selective blocks are get fused. Hence the number of computations are essentially reduced more than 25\%. The pictorial representation of FMI and Entropy are given in Fig.2 and Fig.3 respectively.

TABLE I: Values of Non-Reference Quality Metrics FMI and Entropy

\begin{tabular}{cccc|ccc}
\hline & \multicolumn{3}{c}{ Existing Method } & \multicolumn{3}{c}{ Proposed Method (RBS) } \\
\hline Image Set & No. Of Comp. & FMI & Entropy & No. Of Comp. & FMI & Entropy \\
\hline Set 1 & 256 & 0.9248 & 6.3466 & 207 & 0.9452 & 6.5211 \\
Set 2 & 256 & 0.9127 & 5.7369 & 205 & 0.9315 & 5.7811 \\
Set 3 & 256 & 0.9212 & 5.5746 & 172 & 0.9324 & 5.7158 \\
Set 4 & 256 & 0.9141 & 5.798 & 199 & 0.9315 & 5.9771 \\
Set 5 & 256 & 0.9231 & 5.7145 & 188 & 0.9348 & 5.8185 \\
\hline
\end{tabular}




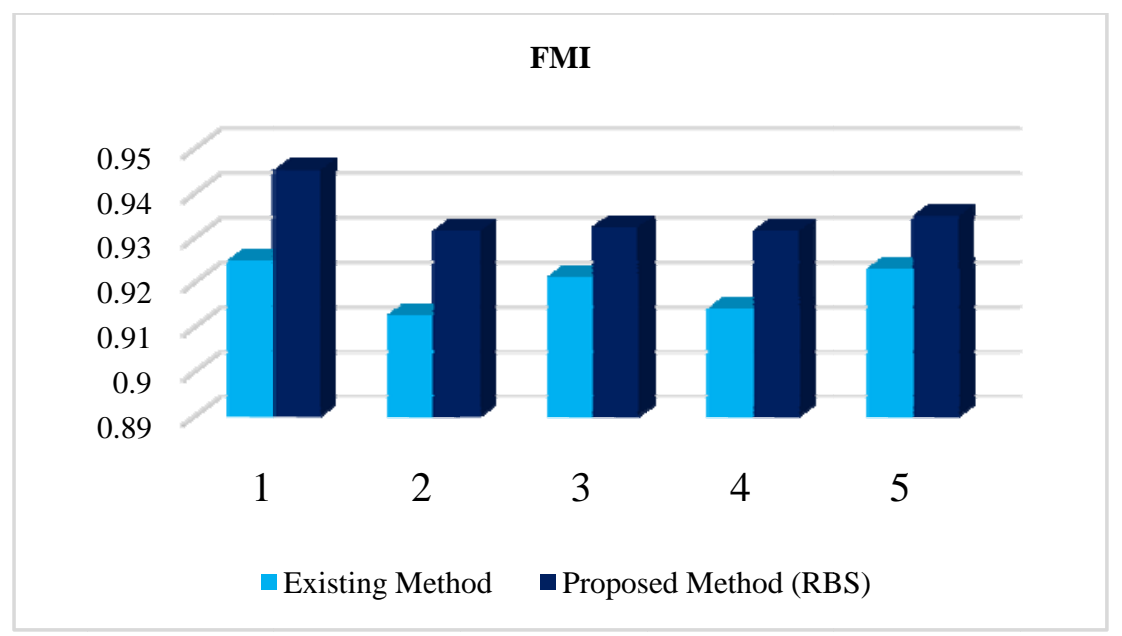

Fig.2: Comparison of FMI values

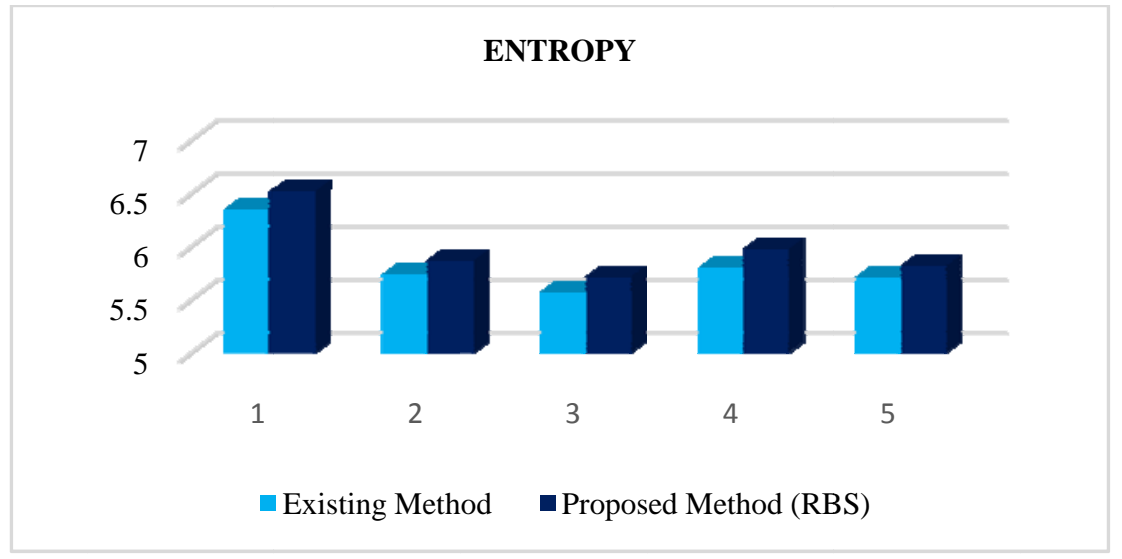

Fig.3: Comparison of FMI values

\section{Peak Signal to Noise Ratio (PSNR)}

PSNR calculates the quality of fused image. The high value represents that how much the fused image is identical to the reference image.

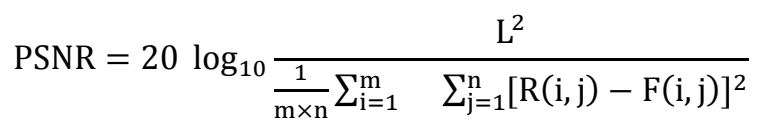

Where $\mathrm{R}$ is the reference image and $\mathrm{F}$ is the fused image

D. Structural Similarity Index Measure (SSIM)

SSIM provides the structural information of the images. It measures the similarity of the fused image and the referenced image.

$$
\operatorname{SSIM}(\mathrm{x}, \mathrm{y})=\frac{\left.\left(2 \mu_{\mathrm{x}} \mu_{\mathrm{y}}+\mathrm{C}_{1}\right)\left(2 \sigma_{\mathrm{xy}}\right)+\mathrm{C}_{2}\right)}{\left(\mu_{\mathrm{x}}^{2}+\mu_{\mathrm{y}}^{2}+\mathrm{C}_{1}\right)\left(\sigma_{\mathrm{x}}^{2}+\sigma_{\mathrm{y}}^{2}+\mathrm{C}_{2}\right)}
$$

The values of performance metrics based on reference image are given in Table II. From the table and graph, it is evident that the proposed method produced good result.

TABLE II: Values of PSNR and SSIM

\begin{tabular}{ccc}
\hline Image Set & PSNR & SSIM \\
\hline Set 1 & 54.5639 & 0.9965 \\
Set 2 & 49.4272 & 0.9975 \\
Set 3 & 55.9436 & 0.9954 \\
Set 4 & 56.7454 & 0.9948 \\
Set 5 & 52.2394 & 0.9918 \\
\hline
\end{tabular}




\section{CONCLUSION}

This paper has proposed a new medical image fusion method. Two different multi modal CT and PET images are fused. CT image provides anatomical structure, whereas PET provides functional information. Hence the two input images are combined to give the maximum information which presented in both images. Contourlet transform with Random block selection (RBS) method has been used and the MAX fusion rule has been used for fusing the coefficients of source images. The resultant fused image helps physicians to diagnose the disease accurately. The quality of fused image has been proved by using the non-reference performance metrics such as FMI and Entropy and the reference based performance metrics such as PSNR and SSIM. In future, instead of selecting the blocks randomly, some criteria are to be used to improve the fusion result.

\section{REFERENCES}

[1] R Indhumathi , Lekshmi P Surendran , KP Indira , Abilash R, “A Literature Review on Image Fusion Techniques for Biomedical Images”, Research Journal of Pharmaceutical, Biological and Chemical Sciences , ISSN: 0975-8585 March-April 2016 RJPBCS 7(2) Page No. 1371

[2] P. Santhi, G. Thirugnanam and P. Mangaiyarkarasi, "Image Fusion Technique for Multi-Resolution Medical Images Using Directional Contourlet Transform”, World Applied Sciences Journal 34 (9): 1177-1182, 2016 ISSN 1818-4952

[3] Shih-GuHuang, "Wavelet for Image Fusion”, Graduate Institute of Communication Engineering and Department of Electrical Engineering, National Taiwan University.

[4] Yong Yang, Song Tong, Shuying Huang and Pan Lin, Log-Gabor Energy Based Multimodal Medical Image Fusion in NSCT Domain-Hindawi Publishing Corporation Computational and Mathematical Methods in Medicine Volume 2014, Article ID 835481, 12 pages

[5] AnuyogamVenkataraman, JavadAlirezaie, Paul Babyn and AlirezaAhmadian, Medical Image Fusion Based on Joint Sparse Method, 2014 Middle East Conference on Biomedical Engineering (MECBME) February 17-20, 2014, Hilton Hotel, Doha, Qatar, 978-1-47994799-7/14/\$31.00 (C2014 IEEE

[6] Sudeb Das and Malay Kumar Kundu, Senior Member, IEEE, A Neuro-Fuzzy Approach for Medical Image Fusion, IEEE Transactions On Biomedical Engineering, VOL. XX, NO. X, MONTH 2013

[7] GuruPrasad S, M.Z. Kurian and H.N. Suma, Fusion of CT and PET Medical Images using Hybrid Algorithm DWT-DCT-PCA, IEEE Explore, Information Science and Security Dec 2015-ISBN 978-1-4673-8611-1

[8] https://en.wikipedia.org/wiki/Contourlet

[9] K. Kalaivani and Y. Asnath Victy Phamila,”Analysis of Image Fusion Techniques based on Quality Assessment Metrics”, Indian Journal of Science and Technology, Vol 9(31), DOI: 10.17485/ijst/2016/v9i31/92553, August 2016

[10] Bagher Akbari Haghighat, Ali Aghagolzadeh, Hadi Seyedarabi, A non-reference image fusion metric based on mutual information of image features, ELSVIER-0045-7906/\$2011, doi:10.1016/j.compeleceng.2011.07.01

\section{AUTHOR PROFILE}

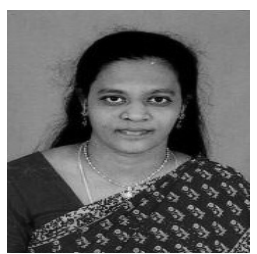

Sheefa Ruby Grace is living in Tirunelveli, Tamilnadu, India. She received her B.Sc., from Madurai Kamaraj University, Madurai. M.Sc. degree from Manonmaniam Sundaranar University, Tirunelveli and M. Phil. degree from Mother Teresa University, Kodaikanal in the Department of Computer Science. From 1996, she worked as a faculty member at Sarah Tucker College, Tirunelveli. Currently she is working for her Ph.D. in Bharathiar University, Coimbatore. Her main research interest is Medical Image Processing.

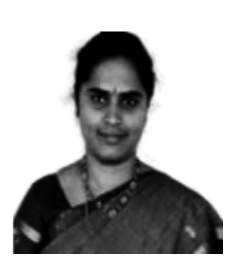

Dr. Marry Immaculate Sheela is living in Ethioppia. She had her PG degree from st. Joseph's college, Bharadhidasan University, Trichy. She got her Ph.D degree on Fusion Algorithms for Intelligent Sensors System in 2008 at Manonmaniam Sundaranar University. She has completed her M.Tech. (CSE) from SRM University, Chennai. Currently she is working as a professor at Dilla University, Dilla. Her main research interests are Sensor Networking, Data Mining, Image Processing. She has published more than 25 papers in International, National and Conference Proceedings. She is the author of three computer science books. She is a recognized research supervisor in reputed universities 